The Clinical Journal of Pain Publish Ahead of Print

DOI:10.1097/AJP.0000000000000309

\title{
The impact of Posttraumatic Stress Disorder on physiological arousal, disability and sensory pain thresholds in patients with chronic whiplash.
}

Rachael L Dunne-Proctor PhD MAPS ${ }^{1,2}$, Justin Kenardy PhD FAPS ${ }^{1,2}$ and Michele Sterling PhD, MPhty BPhty Grad Dip Manip Phty FACP

${ }^{1}$ School of Psychology, The University of Queensland, Australia

${ }^{2}$ Centre of National Research on Disability and Rehabilitation Medicine, The University of Queensland, Australia

${ }^{3}$ Menzies Health Institute of Queensland, Centre of National Research on Disability and Rehabilitation Medicine, NHMRC CRE in Recovery after Road Traffic Injury, Griffith University, Australia. 


\begin{abstract}
Objective: Whiplash associated disorders (WAD) are common and incur substantial personal and economic costs. Research has shown that persistent posttraumatic stress reactions predict poorer functional recovery in WAD; however, the specific mechanism through which this occurs is unclear. The current study is the first to examine the direct impact of PTSD symptoms in WAD using laboratory tested pain measures.
\end{abstract}

Methods: A mixed experimental design was used to examine the impact of exposure to accident cues on chronic WAD individuals with $(n=33)$ and without $(n=39)$ PTSD. Groups were compared at baseline and post accident cue on self-reported pain and negative affect and laboratory tested arousal and sensory pain threshold measures.

Results: At baseline, WAD individuals with PTSD reported greater disability, negative affect, pain, arousal and lower pain thresholds than those without PTSD. As expected, exposure to accident cues resulted in greater increases in arousal and negative affect for those with PTSD. Changes in sensory pain thresholds revealed a hyperalgesic effect in cold pain thresholds for the PTSD group compared to the No PTSD group and mixed findings for pressure pain thresholds.

Discussion: Findings from the current study highlight the negative impact of PTSD on both physical and psychological outcomes in chronic WAD. From a clinical perspective, data suggest that patients exposed to accident-cues may experience arousal that lowers their threshold to certain pain stimuli. Further investigation of effective multidisciplinary interventions and in particular the treatment of PTSD in WAD is identified as an important area of further investigation.

Keywords: Whiplash Associated Disorder, Post-Traumatic Stress Disorder, Motor Vehicle Crash, Pain. 


\section{Introduction}

Whiplash-associated disorders (WADs) are a common, disabling, and costly condition that occurs often as a result of a motor vehicle accident. Current data indicate that up to $50 \%$ of people who experience a whiplash injury will never fully recover ${ }^{1}$ and up to $30 \%$ will remain moderately to severely disabled. ${ }^{2,3}$ The mechanisms underlying persistent pain and disability in this population are poorly understood. ${ }^{4}$

Recent prospective studies have identified persistent posttraumatic stress (PTSD) reactions (distinct from general distress) as an important factor in the development of persistent pain and disability in both acute ${ }^{3,5}$ and chronic WAD. ${ }^{3,6}$ Physical measures of sensory hypersensitity (cold and mechanical hyperalgesia) have been found to predict both moderate to severe PTSD symptoms and more severe pain-related disability at long term follow up. ${ }^{7}$ Current PTSD symptoms have also been found to be associated with reduced activity later in the same day in chronic WAD. ${ }^{8}$ The relationship between pain and PTSD has been widely reported ${ }^{9,10}$ and it has been hypothesised that pain and PTSD may share common neurobiological processes.

McLean and colleagues ${ }^{11}$ propose that abnormal stress system function during and after a stressor may disrupt the neurobiological processes involved in adaptive stress responses and thus increase the risk of an individual's development of both PTSD and pain. Extending and unifying cognitive-behavioural and biological theories, the stress response system is hypothesised as central in the development of abnormal pain and/or emotional processing after a motor vehicle accident, ${ }^{12}$; however, empirical evidence addressing these issues is limited.

While the impact of PTSD symptoms on the experience of pain has begun to be explored in more general chronic pain conditions, empirical data have revealed mixed findings and methodological differences between studies (e.g., assessment measures, inclusion of individuals with heterogeneous pain conditions and PTSD triggers) make it difficult to compare results. ${ }^{13,1415}$ To date, no studies have assessed the direct impact of clinically diagnosed PTSD on the experience of pain. Whiplash Associated Disorders (WAD) provide a specific chronic pain sample where both the injury and traumatic Copyright (C) 2015 Wolters Kluwer Health, Inc. Unauthorized reproduction of the article is prohibited. 
experience have simultaneous onset (although the vulnerabilities to each disorder may exist prior to the accident) and thus provide a good sample in which to explore these relationships.

The aim of the current study is to examine differences in WAD individuals with and without diagnosed accident related PTSD with regard to self-reported pain and negative affect, physiological arousal and laboratory tested sensory pain thresholds. The current study will also be the first to use an experimental design to activate PTSD symptoms through exposure to individually relevant accident cues and examine the impact of this on arousal as well as both self-reported and laboratory tested pain thresholds.

Two specific hypotheses are proposed based on an integration of findings from previous research in heterogeneous pain and trauma samples and WAD specific findings. At baseline, it is predicted that individuals with PTSD will demonstrate greater selfreported disability, pain intensity and negative affect, greater physiological arousal, poorer quality of life and lower sensory pain thresholds than individuals without PTSD (H1). Secondly, it is hypothesised that activation of PTSD symptoms through exposure to an individually relevant accident cue will result in increased negative affect, pain intensity and physiological arousal and lowered sensory pain thresholds (indicating hypersensitivity) in both groups with greater changes evident in the PTSD group compared to the No PTSD group. 


\section{Materials and Method}

\section{Participants}

Seventy-two individuals (47 females) aged 18 to 68 years $(M=34.95, S D=10.02)$ were recruited via advertisements in South-East Queensland. Participants were initially assessed via self-report telephone screening using the Quebec Task Force Classification of WAD. ${ }^{16}$. Eligible participants had chronic WAD (range: 3 to 36 months, $M=$ 30.19months, $S D=18.68$ ) grades II (Neck complaint and musculoskeletal sign(s) (decreased range of motion and point tenderness) or III (Neck complaint and neurological sign(s) (weakness, sensory deficits, decreased or absent deep tendon reflexes). Individuals were excluded if they self-reported (1) any neck fractures of dislocations (classified as WAD IV) (2) experienced a serious head injury or burns (3) have a previous history of whiplash, neck pain or headaches requiring treatment (4) have a diagnosis or are currently receiving treatment for a major psychiatric disorder (e.g. bipolar, schizophrenia etc.) or (5) have insufficient comprehension of English.

\section{Measures}

Background. A background questionnaire was used to collect demographic, accident, injury and current symptom information.

PTSD Diagnosis. The Structured Clinical Interview for DSM-IV TR ${ }^{17}$ is considered a "gold standard" instrument for PTSD diagnosis. For the current study, questions were modified to assess PTSD symptoms related to the motor vehicle crash which caused the whiplash injury and to screen out symptoms attributable to injury, pain, environmental factors or other potentially traumatic events in participants' lives, allowing greater diagnostic accuracy than a self-report questionnaire. Given the overlap in some symptoms between PTSD and WAD (e.g. sleep difficulties, memory, concentration etc.), symptoms which may include both a psychological and pain-related cause were identified and excluded if they were best accounted for by injury or pain.

To be allocated to the PTSD group participants needed to meet criteria for a current diagnosis of PTSD related to the motor vehicle crash which led to the whiplash Copyright (C) 2015 Wolters Kluwer Health, Inc. Unauthorized reproduction of the article is prohibited. 
injury. All other participants (e.g. those not meeting full criteria for PTSD or those meeting criteria for a previous PTSD diagnosis or those with current PTSD symptoms related to other events) were allocated to the No PTSD group.

Self-Report Disability and Quality of Life. The Neck Disability Index (NDI) ${ }^{18}$ percentage score was included as the primary outcome measure for neck pain and disability. The NDI is the most commonly used outcome measure for neck pain, designed particularly for use in patients with whiplash-type injuries and consists of 10 items addressing functional activities such as personal care, lifting, reading, work, driving, sleeping and recreational activities as well as pain intensity, concentration and headache ${ }^{18}$. There are six potential responses to each item ranging from no disability (0) to total disability (5). An overall percentage score (out of 100) is calculated by totalling the responses of each item and multiplying by two. The NDI has been shown to have a high degree of test-retest reliability, internal consistency and validity ${ }^{18}$. A clinically important difference of $7 / 100$ will be used ${ }^{19}$.

The Short Form-36 Health Survey ${ }^{20}$ was included as a measure of generic health status and quality of life. The scales of the SF-36 have been found to be sensitive to clinical manifestations of medical (physical functioning) and global psychiatric (mental health) conditions.

Pain Intensity and Negative Affect. Pain intensity and negative affect were assessed using numerical rating scales (NRS) anchored at zero no pain/distress, 5 somewhat painful/distressing and 10 worst pain imaginable/extremely distressing. Mean negative affect was assessed by averaging the scores of five scales: distress, fear, tension, irritability and sadness. Similar NRS have been used in previous research assessing changes in affect and pain after exposure to accident cues ${ }^{21}$. Pool et al. ${ }^{19}$ reported that clinically important difference on the NRS for patients with neck pain was 2.5.

Physiological Arousal Measures. Heart rate, systolic and diastolic blood pressure were measured as indicators of physiological arousal using the Lifeshirt 200 System (Vivometrics, California). Heart rate was measured via three self-adhering electrodes placed onto the upper check and lateral surface of the abdomen and ECG was sampled at Copyright (C) 2015 Wolters Kluwer Health, Inc. Unauthorized reproduction of the article is prohibited. 
$200 \mathrm{~Hz}$. Blood pressure readings were recorded using the SpaceLabs Ambulatory device.

A control measure of body movement was also taken using an accelerometer and participants were not given any visual or verbal feedback during the study. The data were organised, converted and recorded using Vivo Logic (Vivometrics, California).

Participants were not given any visual or verbal feedback during the experiment. These physiological measures have been previously used in the investigation of posttraumatic stress ${ }^{22}$ and WAD. ${ }^{8}$ The accuracy of the Lifeshirt (Vivometrics) as an ambulatory system compared to standard laboratory-based heart rate monitoring equipment (Biopac) has been demonstrated. ${ }^{23}$

Sensory Pain Thresholds. Qualitative sensory testing was used to measures pain threshold responses to pressure and cold stimuli. These measures have been used extensively in the study of WAD. ${ }^{24}$

Cold pain thresholds were identified a priori as the primary sensory pain threshold measures as previous research has indicated that these measures may be more sensitive to change and have been more clearly linked to PTSD symptoms and poorer recovery in WAD. ${ }^{7}$ Cold Pain Thresholds were measured with the Thermotest System (Somedic AB). The thermode was placed bilaterally on the skin over the cervical spine. From baseline $\left(30^{\circ} \mathrm{C}\right)$ the temperature decreased at a rate of $1{ }^{\circ} \mathrm{C} / \mathrm{s}$ to a minimum of $5^{\circ} \mathrm{C}$. A patientcontrolled switch indicated when participants felt the feeling change from one of cold to one of cold and pain and the temperature at this point was recorded. A mean of three trails were used in analyses ( 10 second pause between measures). Few empirical studies on the psychometric properties of cold pain thresholds exist but research has found strongly correlation with PPTs and have demonstrated adequate reliability ${ }^{25}$. Some normative data are available for comparison, but no studies were found that reported Standard Errors of Measurement to gauge the magnitude of "real change". ${ }^{25}$

Pressure Pain Thresholds (PPTs) were recorded using a digital pressure algometer (Somedic, Sweden), consisting of a $1 \mathrm{~cm}^{2}$ rubber probe connected to a pressure transducer. Pressure was applied at $40 \mathrm{kPa} / \mathrm{s}$ and participants were instructed to press a button when the sensation under the probe changed from one of pressure only to one of pressure and 
pain and the pressure at that point was recorded. PPT's were measured at two cervical spine sites (C2 and C5), a bilateral upper limb site (medial nerve (MN) at the anterior aspect of the elbow) and a bilateral remote site (muscle belly of tibialis anterior (TA)). The mean of three trials at each site (30-second pause between each) were used in analyses. These sites have been previously used in investigation of WAD and have been shown to be reliable and valid. ${ }^{26}$ Local and remote pressure pain thresholds were analyzed separately given the large difference in the standard error of measurement between these sites with values of $144 \mathrm{kPa}$ and above indicating meaningful difference in cervical spine while the standard error for remote sites is estimate to be greater than $243 \mathrm{kPa} .{ }^{26}$

\section{Procedure}

This study was cleared in accordance with the ethical review processes of the University of Queensland and within the guidelines of the National Health \& Medical Research Council. Figure 1 provides details of the procedure and participant flow through the study. Potential participants $(n=156)$ were screened via telephone and eligible participants $(n=92)$ were invited to attend a face-to-face assessment. Written information about the study, a consent form and a pack containing all the self-report questionnaires were sent to eligible participants to complete and return at the face-to-face session, on average one week later.

Seventy-three participants completed the first assessment session. Consent forms and questionnaires were checked for missing data and the SCID was conducted by a registered psychologist with postgraduate clinical training (RD). Participants were then asked to develop an individually relevant accident cue script as previous research has found that personalised scripts are more effective than standard, hypothetical scripts or cues. ${ }^{27}$ This process involved participants writing down the sequence of events for the motor vehicle accident which caused their injury in first-person, present tense and including as much detail as possible about their thoughts, feelings, behaviour, physical responses and sensory experiences. This instruction was intended to facilitate more detailed data-driven processing rather than conceptually driven processing. ${ }^{28}$ A list of possible physical (e.g. heart pounding/racing, muscles tensed, burning sensation, eyes shut, 
nausea etc) and emotional reactions (e.g. sad, angry, fearful, helpless, feeling that things aren't real) was provided to participants to assist with this task and the interviewer provided assistance where required (e.g. clarifying tense, content and detail required). Participants then read the script out loud onto a digital voice recorder. Recordings ranged from 185 to 340 seconds in length with no significant differences between the PTSD $(M=$ 197.45 seconds, $S D=107.23)$ and No PTSD $(M=193.67$ seconds, $S D=133.48)$ groups.

Seventy-two participants completed the second assessment session which was on average one week after the first session. This assessment session was conducted in a climate controlled laboratory and participants completed sensory pain threshold measures in a standardised order (Cold, Pressure (cervical, MN, TA)) based on ease of administration and to measure the primary outcome measures (cold and cervical pressure pain thresholds) as early as practical in the sequence. For bilateral measures left was completed before right. The order of testing was the same for each group so that any order effect of the measures was consistent for both groups and this procedure has been used previously in the study of WAD. ${ }^{25,29}$

Participants were fitted with the Lifeshirt and a five minute relaxation time was used to gain baseline physiological measures, recorded in the final minute. Participants then completed the baseline NRS prior to listening to their individual accident cue via headphones. Participants were instructed to imagine as vividly as possible the accident and imagine their associated physical and emotional reactions. Post-accident cue arousal, NRS and sensory pain threshold measures were completed following the same procedure.

At the completion of the session current pain intensity and affect were reassessed to ensure all participants had returned to baseline levels before exiting the lab. Participants were debriefed as to the aims and rationale for the study, thanked for their time and given a set of contact numbers for any questions or concerns they may have. Participants for whom a diagnosis of PTSD was indicated were also informed about a randomised control trial offering free psychological treatment for PTSD. 


\section{Statistical Analyses}

SPSS for Windows (Version 17) software was used to perform all statistical analyses. The alpha level was set at .05 and Bonferroni corrections were made to maintain this level of Type I error in the context of multiple comparisons. Chi-square and independent groups analysis of variance (ANOVA) were conducted to determine difference between the PTSD and No PTSD groups on demographic variables.

For the cross-sectional comparisons a one-way multivariate analysis of variance (MANOVA) with follow-up univariate comparisons were used to assess differences between the groups on the SF-36 subscales while an independent groups ANOVA were used for the NDI.

A mixed experimental design was used to examine the impact of PTSD on affect, pain intensity, arousal and sensory pain threshold variables at baseline and following direct activation of PTSD symptoms via the accident cue. A combination of $2 \times 2$ mixed ANOVAs and MANOVAs (as specified in the results section), with follow-up univariate analyses were used with PTSD as the between groups variable (PTSD and No PTSD) and Time (Pre- and Post- accident cue) as the within groups variable.

Sensory pain threshold measures were averaged over tree trials at each site. Analyses for PPTs at local (cervical (C2, C5)) and remote (MN, TA) sites were conducted separately given the large differences in the standard error of measurement in these sites (see Measures sections for greater detail). Independent samples t-tests revealed no significant difference between the left and right sides for any of the bilateral sensory measures $(p>.05)$; therefore, the mean values were used in further analyses (similar protocol have been observed in previous research WAD. ${ }^{30}$

Power analyses were conducted prior to the commencement of the study to determine the number of participants required in each group to allow adequate power to detect meaningful differences (as defined for each measure in the Methods section) both within and between groups. In accordance with guideline set out by Cohen, ${ }^{31}$ power was set at .80 and the alpha level was .05 . Given the limited previous empirical research on the 
research with regard to general chronic pain, general PTSD and where available studies looking specifically at whiplash and trauma symptoms. Effect sizes varied greatly between studies depending on the sample and measures used. Moderate to large effect sizes were estimated for between groups (PTSD/No PTSD) differences on self-reported whiplash pain, ${ }^{7}$ quality of life ${ }^{32}$ and physiological measures. ${ }^{33,34}$ Moderate effect sizes were estimated for within group differences (baseline and post-exposure) in arousal and selfreported pain. ${ }^{21}$ Estimates for changes in sensory pain thresholds between and within subjects were determined to be small to moderate based on previous research ${ }^{35}$ and preliminary laboratory data. Results of the various power analyses were collated and it was determined that a minimum of 34 participants in each group would be required to allow enough power to detect meaningful differences (based on the clinically important change values for each measure outlined in the Measures section) both between and within groups. A total of 72 participants were recruited to the study and it was determined that this would allow sufficient power to conduct the planned analyses.

\section{Results}

\section{PTSD Diagnosis}

Based on the SCID, 33 participants (45.8\%) were diagnosed with current motor vehicle crash related PTSD while 39 (54.2\%) did not meet full criteria for a current diagnosis. Of note, seven participants in the No PTSD group (17.9\%) did meet criteria for partial PTSD (i.e. met criteria for re-experiencing symptoms and either avoidance or increased arousal symptoms but not all diagnostic criteria).

Six participants from the PTSD group (18.2\%) met criteria for lifetime diagnosis of PTSD pre-dating the motor vehicle crash while 13 participants (33.3\%) from the No PTSD group met criteria for lifetime PTSD. Chi squared analyses revealed no significant differences between the groups in the frequency of lifetime $\operatorname{PTSD}\left(\chi^{2}(1)=2.11, p=.15\right)$ or number of trauma exposures (see Table 1). With respect to the nature of prior trauma, the PTSD group differed from the No PTSD group only in exposure to a serious accident, fire or explosion $\left(\chi^{2}(1)=17.44, p>.01\right)$ and physical assault by a stranger $\left(\chi^{2}(1)=9.49, p\right.$ $>$.01) while exposure to all other specified traumas was equivalent (see Table 1). 


\section{Cross Sectional Analyses}

Demographic Analyses. Table 1 displays the demographic, injury and trauma exposure characteristics for PTSD and No PTSD Groups. Chi squared analyses revealed no significant differences between the groups on demographic (gender, education, work status) or medical variables (initial WAD symptoms, current symptoms, other medical conditions ( $42.4 \%$ and $41.0 \%$ respectfully) or other injuries (48.5\% and 38.5\% respectfully)). A one-way MANOVA also revealed no significant differences between the groups with regard to age, height and weight. One-way ANOVA analyses revealed no difference between groups in time since the accident; however, participants with PTSD did report more sites of pain than those without PTSD $(t(70)=-2.37, p=.02)$. Follow-up univariate analyses revealed individuals with PTSD were more likely to report pain in their $\operatorname{back}\left(\chi^{2}(1)=4.09, p=.04\right)$ and shoulders $\left(\chi^{2}(1)=6.29, p=.01\right)$, with no differences in arm and leg pain.

Comparison of pain, disability and quality of life. There was no difference between the PTSD group $(M=41.69, S D=13.90)$ and No PTSD group $(M=35.69, S D=$ 12.18) on the primary outcome of NDI. This difference approached but was not statistically $(t(70)=-1.95, p=.06$, Cohen's $d=0.46)$ or clinically significant (mean difference $>7 / 100)$.

Table 2 displays the means and effect sizes for SF-36 scores between the PTSD and No PTSD groups. A MANOVA revealed that the PTSD group reported significantly poorer quality of life (Wilk's lambda $\left.=.69, F(11,60)=2.49, p=.01, \eta_{\mathrm{P}}^{2}=.31\right)$ compared to the No PTSD group. Follow-up univariate analyses revealed significant differences on the subscales of Bodily Pain $(F(1,70)=5.20, p=.03)$, General Health $(F(1,70)=8.01, p$ $=.006)$, Social Functioning $(F(1,70)=9.95, p=.002)$ and Mental Health $(F(1,70)=$ $19.41, p<.001)$. 


\section{Experimental Analyses}

An experimental design was used to examine the impact of PTSD on affect, pain intensity, arousal and pain threshold variables at baseline and following direct activation of PTSD symptoms via the accident cue. Separate mixed models ANOVAs were used for each outcome variable with PTSD as the between groups variable (PTSD and No PTSD) and Time as the within groups variable (Pre- and Post- accident cue). The alpha level was set at .05 and Bonferroni corrections were applied to adjust for multiple comparisons resulting in $p=.01$ criterion for these analyses.

Self-Reported NRS Negative Affect. The PTSD group demonstrated significantly higher negative affect at both baseline and post accident cue $(F(1,70)=15.91, p<.001$, $\left.\eta_{\mathrm{P}}^{2}=.19\right)$ and both groups demonstrated an increase in negative affect following the trauma-cue $\left(F(1,70)=61.47, p<.001, \eta_{\mathrm{P}}^{2}=.26\right)$. (Figure 2). A significant Time $\mathrm{x}$ PTSD interaction was also found $\left(F(1,70)=25.15, p=.001, \eta_{\mathrm{P}}^{2}=.26\right)$ with stronger increases in negative affect in the PTSD group $(\mathrm{T} 1=3.16, \mathrm{~T} 2=6.02)$ compared to the No PTSD group $(\mathrm{T} 1=1.8, \mathrm{~T} 2=2.42)$ from baseline to post accident cue. With regard to clinical significance, the difference between groups at baseline were comparable while changes between and within groups following the trauma cue were considered clinically significant (difference $>2.5$ ).

Self-reported NRS Pain Intensity. For NRS pain intensity at baseline and following the accident cue, main effects for $\operatorname{PTSD}\left(F(1,70)=8.63, p=.004, \eta_{\mathrm{P}}^{2}=.11\right)$, Time $\left(F(1,70)=40.63, p<.001, \eta_{\mathrm{P}}^{2}=.37\right)$ and a significant Time $\mathrm{x}$ PTSD interaction $(F$ $\left.(1,70)=29.56, p<.001, \eta_{\mathrm{P}}^{2}=.30\right)$ were found. The PTSD group reported stronger increases in pain intensity after the accident cue $(\mathrm{T} 1=3.58, \mathrm{~T} 2=4.55)$ compared to the No PTSD group $(\mathrm{T} 1=2.82, \mathrm{~T} 2=2.90)$. Figure 3 . Interestingly, none of the changes in selfreported pain intensity between or within groups met criteria for clinical significance (difference $>2.5$ ). 
Arousal Measures. A summary of the means and standard deviations for the physiological arousal measures are presented in Table 3. A repeated measures MANOVA revealed significant main effects for $\operatorname{PTSD}\left(F(3,68)=7.20, \mathrm{p}<.001, \eta_{\mathrm{P}}^{2}=.24\right)$, Time $(F$ $\left.(3,68)=52.73, \mathrm{p}<.001, \eta_{\mathrm{P}}^{2}=.70\right)$ and a significant Time $\mathrm{x}$ PTSD interaction $(F(3,68)=$ $8.33, p<.001, \eta_{\mathrm{P}}^{2}=.27$ ) with greater increases in arousal seen in the PTSD group compared to the No PTSD group following the accident cue.

Sensory Pain Threshold Measures. A summary of the means and standard deviations for the sensory pain threshold measures are also presented in Table 3.

For the primary outcome measure of cold pain threshold, there was a significant main effect for $\operatorname{PTSD}\left(F(1,70)=10.02, p=.002, \eta_{\mathrm{P}}^{2}=.13\right)$ with the PTSD group demonstrating lower thresholds across time than the No PTSD group. No significant main effect for Time was found $\left(F(1,70)=0.69, p=.41, \eta_{\mathrm{P}}^{2}=.01\right)$; however, there was a significant Time x PTSD interaction $\left(F(1,70)=8.55, p=.005, \eta_{\mathrm{P}}^{2}=.10\right)$. As seen in Table 3, the PTSD group showed a significant decrease in their threshold to cold temperature from pre- to post- trauma cue while there was minimal change for the No PTSD group over time.

For the cervical pressure pain, a significant main effect for $\operatorname{PTSD}(F(2,67)=$ $\left.11.10, p<.001, \eta_{\mathrm{P}}^{2}=.25\right)$ revealed lower PPTs in the cervical spine for the PTSD group compared to the No PTSD group at both baseline and post accident cue. There was no main effect for Time found $\left(F(2,67)=.84, p=.44, \eta_{\mathrm{P}}^{2}=.02\right)$. Table 3 shows that the PTSD group demonstrated a trend towards greater decrease in cervical PPTs after the trauma cue compared to the No PTSD group; however, when the Bonferroni correction was applied the Time $\mathrm{x}$ PTSD interaction did not reach significance $(F(2,67)=3.13, p=$ $\left..05, \eta_{\mathrm{P}}^{2}=.09\right)$. Despite the between groups analyses reaching statistical significance, the difference between the PTSD and No PTSD groups at each time point were not considered clinically significance (defined in the Measures section as $>144 \mathrm{kPa}$ ). 
For the remote site PPTs, no main effect for Time $\left(F(2,69)=.50, p=.61, \eta_{\mathrm{P}}^{2}=\right.$ $.01)$ or the Time $x$ PTSD interaction were found $\left(F(2,69)=1.37, p=.26, \eta_{\mathrm{P}}^{2}=.04\right)$. Table 3 displays a trend towards lower pressure pain thresholds in remote sites for the PTSD group compared to the No PTSD group across time; however, when the Bonferroni correction was applied this main effect for PTSD was not significant $(F(2,69)=3.95, p=$ $\left..02, \eta_{\mathrm{P}}^{2}=.10\right)$ and this difference was also not determined to be clinically significant (defined as $>243 \mathrm{kPa})$.

Correlations between changes in outcome measures. Correlations between percent changes in outcome measures in PTSD group are illustrated in Table 4.

\section{Discussion}

The results of this study support and extend on previous findings that individuals with chronic whiplash, particularly those with comorbid PTSD, present with variable and complex patterns of physical, psychological and sensory impairments. ${ }^{25,29}$ The primary purpose of this study was to examine the impact of comorbid PTSD in individuals with chronic whiplash on disability and quality of life, physiological arousal and sensory pain thresholds. The secondary aim was to assess whether PTSD exerted a general effect and or a specific effect on these outcomes through direct activation of PTSD symptoms via accident cues.

Findings from the cross-sectional analysis indicate that in general, individuals with chronic WAD and PTSD reported greater pain locations, general disability and poorer quality of life compared to WAD individuals without PTSD. While there was a moderate trend for individuals with PTSD to report greater neck pain and disability this was not statistically or clinically significant in the current sample. These findings are consistent with previous longitudinal research findings that baseline PTSD symptoms predicted the persistence and severity of WAD symptoms and disability at 6 and 12 months follow-up. ${ }^{3,6}$ More generally, these results are also consistent with previous cross-sectional findings that chronic pain patients with comorbid PTSD experience more intense pain and affective distress ${ }^{36}$ and greater disability ${ }^{32,37}$ than pain patients without PTSD.

Copyright (C) 2015 Wolters Kluwer Health, Inc. Unauthorized reproduction of the article is prohibited. 
This was the first empirical study to specifically activate PTSD symptoms in patients with chronic WAD and assess changes in self-reported and laboratory tested pain using an experimental design. The results support previous general chronic pain research findings that individuals with PTSD reported greater negative affect ${ }^{21}$ and arousal at baseline ${ }^{34}$ and that the activation of PTSD symptoms resulted in further affective and physiological reactivity. ${ }^{27}$ In the current study, individuals with PTSD were also found to have lower sensory pain thresholds compared to those without PTSD across cold and cervical pressure pain measures at baseline and post-trauma cue. This difference between the groups was observed in the remote (arm and leg) locations; however, when an adjustment for multiple comparisons was applied this no longer reached significance and these differences were not considered clinically significant. While generalized central nervous system hyperexcitability has been consistently reported in WAD, ${ }^{4}$ the finding of site specific hyperalgesia between the PTSD and No PTSD groups in current study is in line with previous conclusions ${ }^{35}$ that sensory hypersensitivity cannot be attributed to psychological factors alone and may reflect the contribution of other neurobiological changes or a complex interplay between these substrates.

Interestingly, exposure to the accident cue in the current study resulted in further significant decreases in cold pain thresholds at the site of injury while minimal changes were found in local or remote pressure pain thresholds between or across groups. The hyperalgesic effect demonstrated in the cold pain thresholds is contrary to some earlier research which has suggested an analgesic effect of trauma exposure ${ }^{13} 38$ However, this finding is consistent with research demonstrating lower pain thresholds after laboratory induced anxiety in healthy controls ${ }^{14}$ and in line with studies demonstrating lower baseline pain thresholds in whiplash patients ${ }^{25}$ and patients with heterogeneous chronic pain and posttraumatic stress symptoms. ${ }^{37}$

It should be noted that a conservative approach to managing type I error in the context of multiple comparisons was taken in the current study. This is considered a strength in that it maintains a lower study-wide error rate given the number of comparisons performed; however, the deflated error rate for each analyses also increases the type II error rate. ${ }^{39}$ As a result comparisons with a slightly smaller (but potentially meaningful Copyright (C) 2015 Wolters Kluwer Health, Inc. Unauthorized reproduction of the article is prohibited. 
and important) effect sizes which would have previously been considered statistically significant were deemed non-significant. In particular this applied to the difference between PTSD and No PTSD groups for remote pressure pain sites and also to the interaction between PTSD group and Time (pre and post accident cue) for the cervical spine pressure pain threshold which would be consistent with the cold pain threshold findings as previously discussed.

The specific findings of cold hyperalgesia is also consistent with previous research indicating that cold pain thresholds are more likely than other sensory thresholds measures to be associated with poorer outcomes for WAD ${ }^{25}$ and this may reflect the mechanism through which neurobiological changes in more complex cases of WAD may occur. Previous research has reported lower pain threshold, lower b-endorphin levels, and decreased production and release of methionine enkephalin and stress-induced analgesia in individuals with PTSD. ${ }^{13,40}$

The findings that exposure to an individually relevant accident cue resulted in changes in cold pain thresholds may also reflect altered attentional or emotional responses or changes in the interpretation of painful stimuli in the PTSD group. An alternative, but not mutually exclusive explanation is that altered sensory processing occurs following activation of the stress system through exposure to the accident cue. ${ }^{38}$ Previous research has identified the ventral basal ganglia dopamine neurotransmitter system being involved in stress response, negative emotion, and pain regulation, ${ }^{15}$ suggesting that pain, negative affect and stress associated with the response to trauma may share similar neurobiological mechanisms. Results from the current study provide additional support that these systems and responses may be related; however, determination of the underlying mechanisms awaits further investigation.

The mixed findings of the current study again indicate that psychological factors may play a role but are the only or main factors responsible for central hyper-excitability in patients with WAD. ${ }^{35}$ Indeed, in a recent pilot study looking at the impact of PTSD treatment in WAD patients with comorbid PTSD, hyperalgesia and disruption to the sensory nervous system remained despite the alleviation of PTSD symptoms, ${ }^{41}$ 
highlighting the need for multidisciplinary approaches to further investigate this comorbidity.

A major strength of the current study is the use of a clinical interview to differentiate between the overlap of symptoms between PTSD and pain. However, it must also be noted that using this categorical method for comparison of those with PTSD and those with No PTSD also results in a proportion of participants in the No PTSD group (18\%) meeting criteria for partial PTSD. Thus, the effect sizes demonstrated in the current study are conservative and may in fact be minimising the true difference between the groups. Future research utilising a larger sample size may investigate the relationship using a third partial or sub-threshold PTSD group to allow more clear conclusions regarding the importance of diagnostic classification or symptom severity with regard to outcomes.

A further strength of the current study is the use of both self-reported and laboratory tested pain measures allowing different aspects of pain to be explored. A limitation of this process; however, is the inherent difficulty in measuring pain realistically in an experimental environment. In this study participants were aware of the cause, duration and lack of tissue damage that would result from the pain induction and had control to request the cessation of the stimuli compared to experiences outside the laboratory where pain may be perceived as more threatening, impacting on ratings of intensity and affective distress. As highlighted by Stone and colleagues ${ }^{24}$ the use of sensory pain threshold measures requires further research to determine more concisely the stimulus parameters and body sites which are most reliable and valid.

In addition, the potential confound that the pain induction process itself being an accident cue was considered. Previous research has reported that the pain induction task itself had little effect on affective distress, avoidance or physiological reactivity regardless of PTSD diagnosis ${ }^{21}$ and attempts to control for this was made through assessing baseline measures of physiological arousal and self-reported pain and affect after the baseline measures of sensory pain thresholds to provide a more conservative baseline measure.

It should also be noted that the potential impact of participants engaging in various coping strategies and reactions to the accident cue (i.e. flashbacks, dissociation, use of Copyright (C) 2015 Wolters Kluwer Health, Inc. Unauthorized reproduction of the article is prohibited. 
distraction strategies) despite standardised instructions is also noted with different responses likely to influence physiological reactivity. ${ }^{42}$ The current study did not differentiate between the presence of these different coping strategies and this represents an avenue for further inquiry. As engaging in exposure tasks in inherently difficult for individuals with PTSD, future research would also benefit from more detailed assessment of individuals ability and willingness to engage in this task and measurement to validate this experimental manipulation.

The findings of the current study further highlight the negative impact of PTSD on both physical and psychological outcomes in WAD and the need for further investigation into effective treatments for this comorbidity. From a clinical perspective, these data suggest that patients exposed to accident cues may experience anxiety that lowers their threshold to certain pain stimuli, particularly at the site of the injury. Results of the current study and previous empirical and theoretical studies suggest that biological, psychological and physical aspects of both PTSD and chronic pain play a role in the development and maintenance of this comorbidity. 


\section{References}

1. Carroll L, Holm L, Hogg-Johnson S, et al. Course and Prognostic Factors for Neck Pain in Whiplash-Associated Disorders (WAD). Results of the Bone and Joint Decade 2000-2010 Task Force on Neck Pain and Its Associated Disorders. Spine 2008;33:583-92.

2. Rebbeck T, Sindhausen D, Cameron I. A prospective cohort study of health outcomes following whiplash associated disorders in an Australian population. Injury Prevention 2006;12:86-93.

3. Sterling M, Jull G, Vicenzino B, Kenardy J, Darnell R. Physical and psychological factors predict outcome following whiplash injury. Pain 2005;114:141-8.

4. Curatolo M, Sterling M. Pain-processing mechanisms in whiplash associated disorders. In: Sterling M, Kenardy J, eds. Whiplash: evidence-base for clinical practice Australia: Elsevier; 2011.

5. Drottning M, Staff P, Levin L, Malt U. Acute emotional response to common whiplash predicts subsequent pain complaints: a prospective study of 107 subjects sustaining whiplash injury. Nordic Journal of Psychiatry 1995;49:293-9.

6. Buitenhuis J, DeJong J, Jaspers J, Groothoff J. Relationship between posttraumatic stress disorder symptoms and the course of whiplash complaints. Journal of Psychosomatic Research 2006;61:681-9.

7. Sterling M, Hendrikz J, Kenardy J. Similar factors predict disability and PTSD trajectories following whiplash injury. Pain 2011;152:1272-8.

8. Sterling M, Chadwick B. Psychological Processes in Daily Life With Chronic Whiplash: Relations of post-traumatic stress symptoms and fear-of-pain to hourly pain and uptime. Clinical Journal of Pain 2010;26:573-82.

9. Otis JD, Gregor K, Hardway C, Morrison J, Scioli E, Sanderson K. An examination of the co-morbidity between chronic pain and posttraumatic stress disorder on US Veterans. Psychological Services 2010;7:126.

10. Scioli-Salter ER, Forman DE, Otis JD, Gregor K, Valovski I, Rasmusson AM. The Shared Neuroanatomy and Neurobiology of Comorbid Chronic Pain and PTSD: Therapeutic Implications. The Clinical Journal of Pain 2015;31:363-74.

11. McLean S, Clauw D, Abelson J, Liberzon I. The development of persistent pain and psychological morbidity after motor vehicle collision: intergrating the potential role of stress response systems into a biopsychosocial model. Psychosomatic Medicine 2005;67:783-90.

12. Pitman RK, van der Kolk BA, Orr SP, Greenberg MS. Naloxone-reversible analgesic response to combat-related stimuli in posttraumatic stress disorder. A pilot study. Arch Gen Psychiatry 1990;47:541-4.

13. Friedman MJ. What might the psychobiology of posttraumatic stress disorder teach us about future approaches to pharmacotherapy? The Journal of clinical psychiatry 2000;61 Suppl 7:44-51.

14. Rhudy J, Meagher M. Fear and anxiety: divergent effects on human pain thresholds. Pain 2000;84:65-75.

15. Scott DJ, Heitzeg MM, Koeppe RA, Stohler CS, Zubieta JK. Variations in the human pain stress experience mediated by ventral and dorsal basal ganglia dopamine activity. J Neurosci 2006;26:10789-95.

16. Spitzer W, Skovron M, Salmi L, et al. Scientific Monograph of Quebec Task Force on Whiplash associated Disorders: redefining "Whiplash" and its management. Spine 1995;20:1-73.

17. First M, Spitzer R, et al. Structured Clinical Interview for DSM-IV Axis Disorders, Research Version, Patient Edition. New York: Biometrics Research; 1995.

18. Vernon H, Mior S. The Neck Disability Index: A study of reliability and validity. Journal of Manipulative Physiological Therapeutics 1991;14:409-15.

19. Pool J, Ostelo R, Hoving J, Bouter L, de Vet H. Minimal clinically important change of the neck disability index and the numerical rating scale for patients with neck pain. Spine 2007;32:3047-51.

20. Ware J, Snow K, Kosinski M, Gandek B. SF-36 health survey: manual and interpretation guide. Boston, MA: The Health Institute; 1993.

21. Spertus I. Posttraumatic stress disorder and chronic pain: Fear activation in response to a pain stimulus2001.

Copyright (C) 2015 Wolters Kluwer Health, Inc. Unauthorized reproduction of the article is prohibited. 
22. Beckham J, Taft C, Vrana S, et al. Ambulatory monitoring and physical health reportin vietnam veterens with and without chronic posttraumatic stress disorder. Journal of Traumatic Stress 2003;16:329-35.

23. Heilman KJ, Porges SW. Accuracy of the LifeShirt (Vivometrics) in the detection of cardiac rhythms. Biol Psychol 2007;75:300-5.

24. Stone A, Vicenzino B, Lim E, Sterling M. Measures of central hyperexcitability in chronic whiplash associated disorder - A systematic review and meta-analysis. Manual Therapy 2012;18:111-7.

25. Sterling M, Jull G, Vicenzino B, Kenardy J. Sensory hypersensitivity occurs soon after whiplash injury and is associated with poor recovery. Pain 2003;104:509-17.

26. Sterling M, Carllson Y, Crommert L, Jull G. Are cervical physical outcome measures influenced by the presence of symptomatology? Physiotherapy Research International 2002;7:113-21.

27. Orr SP, Pitman RK. Psychophysiologic assessment of attempts to simulate posttraumatic stress disorder. Biol Psychiatry 1993;33:127-9.

28. Ehlers A, Clark DM. A cognitive model of posttraumatic stress disorder. Behav Res Ther 2000;38:319-45.

29. Sterling M, Jull G, Kenardy J. Physical and psychological predictors of outcome following whiplash injury maintain predictive capacity at long term follow-up. Pain 2006;122:102-8.

30. Sterling M. Testing for sensory hypersensivity or central hyperexcitability associated with cervical spine pain. Journal of Manipulative and Physiological Therapeutics 2008;31:534-9.

31. Cohen J. A power primer. Psychol Bull 1992;112:155-9.

32. Sherman JJ, Turk DC, Okifuji A. Prevalence and impact of posttraumatic stress disorder-like symptoms on patients with fibromyalgia syndrome. Clin J Pain 2000;16:127-34.

33. Cohen H, Neumann L, Shore M. Autonomic dysfunction in patients with fibromyalgia: Application of power spectral analysis of heart rate variability. Semin Arthritis Rheum 2000;29:217-27.

34. Shalev A, Orr S, Pitman R. Psychophysiologic assessment of traumatic imagery in Israeli civilian patients with posttraumatic stress disorder. American Journal of Psychiatry 1993;150:620-4.

35. Sterling M, Pettiford C, Hodkinson E, Curatolo M. Psychological factors are related to some sensory pain thresholds but not nociceptive flexion reflex threshold in chronic whiplash. Clinical Journal of Pain 2008;24:124-30.

36. Toomey T, Seville J, Abashian S, Finkel A, Mann J. Circumstances of chronic pain onset: relationship to pain description, coping and psychological distress. American Pain Society1994.

37. Beckham JC, Crawford AL, Feldman ME, et al. Chronic posttraumatic stress disorder and chronic pain in Vietnam combat veterans. J Psychosom Res 1997;43:379-89.

38. Defrin R, Ginzburg K, Solomon Z, et al. Quantitative testing of pain perception in subjects with PTSD - Implications for the mechanism of the coexistence between PTSD and chronic pain. Pain 2008;138:450-9.

39. Perneger TV. What's wrong with Bonferroni adjustments. Bmj 1998;316:1236-8.

40. Schwartz AC, Bradley R, Penza KM, et al. Pain medication use among patients with posttraumatic stress disorder. Psychosomatics 2006;47:136-42.

41. Dunne R, Kenardy J, Sterling M. A randomised controlled trial of cognitive behavioural therapy for the treatment of PTSD in the context of chronic Whiplash. Clinical Journal of Pain 2012;28:755-65.

42. Lanius RA, Williamson PC, Bluhm RL, et al. Functional connectivity of dissociative responses in posttraumatic stress disorder: a functional magnetic resonance imaging investigation. Biol Psychiatry 2005;57:873-84. 


\section{Table 1}

Demographic, Injury and Trauma Exposure Characteristics for PTSD and No PTSD Groups

$\begin{array}{lc}\text { PTSD } & \text { No PTSD } \\ (n=33) & (n=39)\end{array}$

Months since the accident

$26.15(14.92)$

$32.21(19.07)$

Highest Education \%

- Year 10 or below

- Senior high school

18.2

- Diploma/trade certificate

30.3

- Degree

Height (centimeters)

Weight (kilograms)

72.86

Work Status \%

- Employed

- home duties

- unemployed/retired

\section{Initial Symptoms}

Neck pain began $\%$

- Immediately

- $\quad$ within 24 hours

- $\quad$ after 24 hours

Immediate neck movement restriction \%

- not at all

- mild

- moderate

- severe
0

36.4

18.2

45.5

75.8

51.5

$2.55(.90)$

100
12.8

25.6

25.6

35.9

84.6

53.8

$2.10(.68)$

100

Copyright (C) 2015 Wolters Kluwer Health, Inc. Unauthorized reproduction of the article is prohibited. 
- Back

- Shoulders

- Arms

- Legs

\section{Previous Trauma Exposure}

Number of prior traumatic exposures

Reported previous exposure to: \%

Accident, fire or explosion

Natural disaster

Physical assault, known perpetrator

Physical assault, unknown perpetrator

Sexual assault, known perpetrator

Sexual assault, unknown perpetrator

- $\quad$ Military combat

Sexual contact $(<18 y r s)$ with person $>5$ yrs older

- $\quad$ Imprisonment

- $\quad$ Torture

Life-threatening illness

Other trauma
87.9

59.0

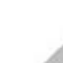

0 


\section{Table 2}

Means (Standard Deviations) and Cohen's d Statistics of the SF-36 Health Survey for PTSD and No PTSD Groups

\begin{tabular}{lccc}
\hline & \multicolumn{1}{c}{ PTSD } & No PTSD & Cohen's d \\
& $(\boldsymbol{n}=\mathbf{3 3})$ & $(\boldsymbol{n}=\mathbf{3 9})$ & \\
\hline Physical functioning & $57.88(27.10)$ & $62.18(19.02)$ & 0.18 \\
Physical role & $21.21(31.32)$ & $37.18(37.99)$ & 0.46 \\
Bodily Pain & $34.15(15.72)$ & $44.05(19.13)$ & 0.57 \\
General Health & $50.06(20.15)$ & $60.69(16.11)$ & $0.58^{*}$ \\
Vitality & $33.79(20.27)$ & $43.84(22.11)$ & 0.47 \\
Social functioning & $47.12(17.67)$ & $62.00(21.04)$ & $0.77^{*}$ \\
Emotional role & $40.39(39.81)$ & $58.95(42.94)$ & 0.45 \\
Mental Health & $54.30(16.00)$ & $69.74(16.10)$ & $0.96^{* *}$ \\
Health transition & $39.39(28.66)$ & $46.80(20.02)$ & 0.30 \\
Phys Health Component & $39.55(17.70)$ & $49.72(13.92)$ & $0.64^{*}$ \\
Mental Health Component & $45.06(18.03)$ & $59.03(16.47)$ & $0.81^{* *}$ \\
\hline$*<.01, * * p<.001$ & & & \\
\hline
\end{tabular}




\section{Table 3}

Means (Standard Deviations) for Physiological Arousal and Sensory Pain

Threshold Measures from Baseline to Post- Trauma Cue for PTSD and No PTSD

Groups

\begin{tabular}{|c|c|c|c|c|}
\hline & \multicolumn{2}{|c|}{ Baseline } & \multicolumn{2}{|c|}{ Post-Exposure } \\
\hline & PTSD $(n=33)$ & No PTSD $(n=39)$ & PTSD $(n=33)$ & No PTSD $(n=39)$ \\
\hline Systolic BP & $124.42(11.60)$ & 119.84 (11.07) & $128.73(12.73)$ & $20.64(12.59)$ \\
\hline Diastolic BP & $77.78(6.98)$ & $75.79(8.76)$ & $83.67(9.49)$ & 6.30) \\
\hline Heart Rate & $74.93(10.21)$ & $68.27(4.98)$ & 80.30 & \\
\hline Pressure Pain - MN & $161.78(58.76)$ & $198.24(90.19)$ & & $204.32(89.42)$ \\
\hline - TA & $298.99(103.36)$ & $394.00(181.43)$ & $01(128.78)$ & 408.09 199.17) \\
\hline$-\mathrm{C} 2$ & $157.41(76.78)$ & $223.52(87.09)$ & & $228.56116 .00)$ \\
\hline$-\mathrm{C} 5$ & $151.53(67.79)$ & $222.15(89.61)$ & $130.89(69.16)$ & $234.13(104.19)$ \\
\hline Cold Pain & $13.93(5.61)$ & $10.15(6.82)$ & $5.10(6.40)$ & $9.50(6.89)$ \\
\hline
\end{tabular}

Note. $\mathrm{BP}=$ Blood Pressure; $\mathrm{MN}=$ Median Nerve; $\mathrm{TA}=$ Tibialis Anterior; $\mathrm{C} 2$ = second cervical spinous process; $\mathrm{C} 5=$ fifth cervical spinous process 
Table 4. Correlations between percent change in outcome measures for PTSD group.

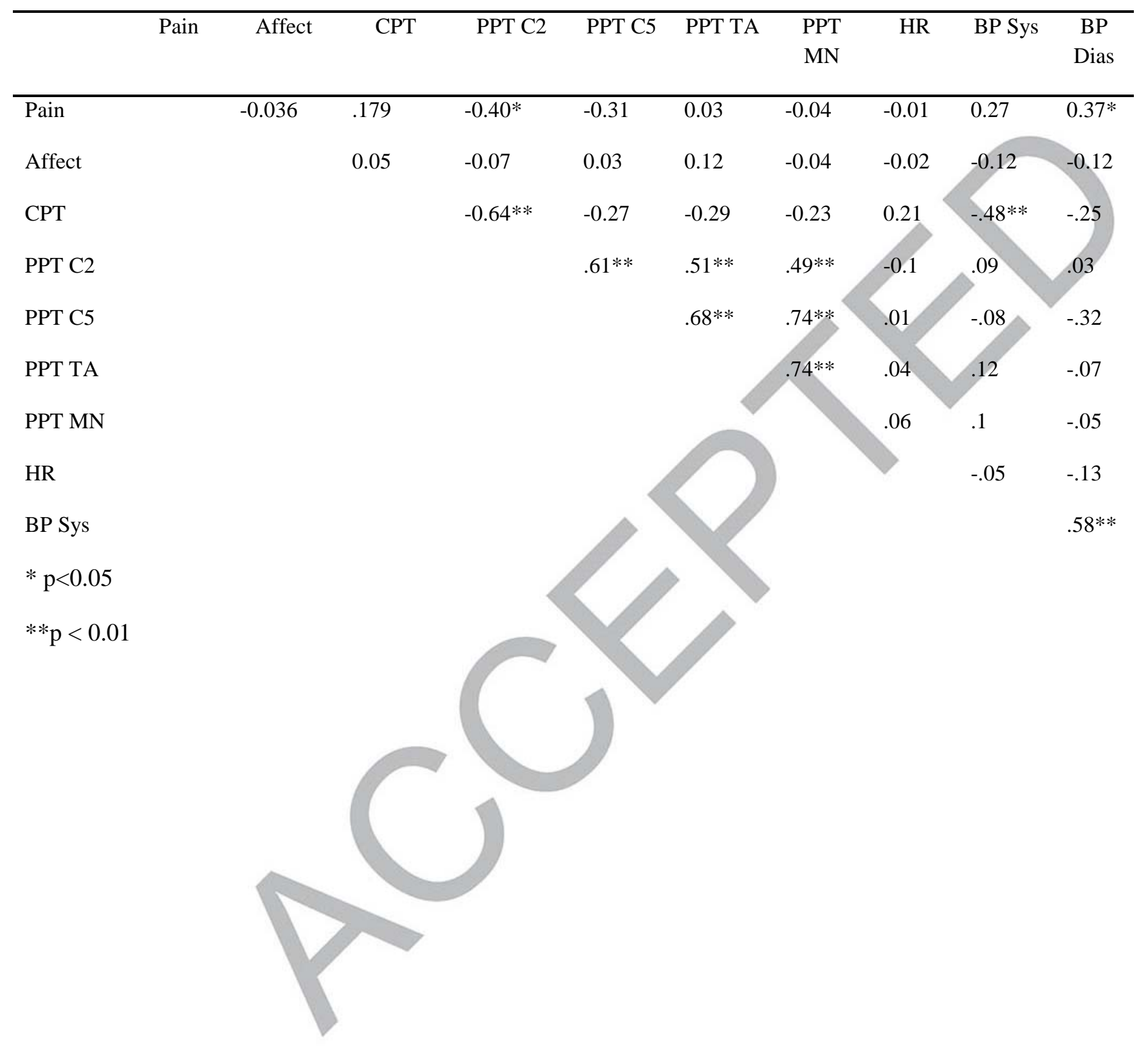


Figure 1. Flow Diagram for the Procedure

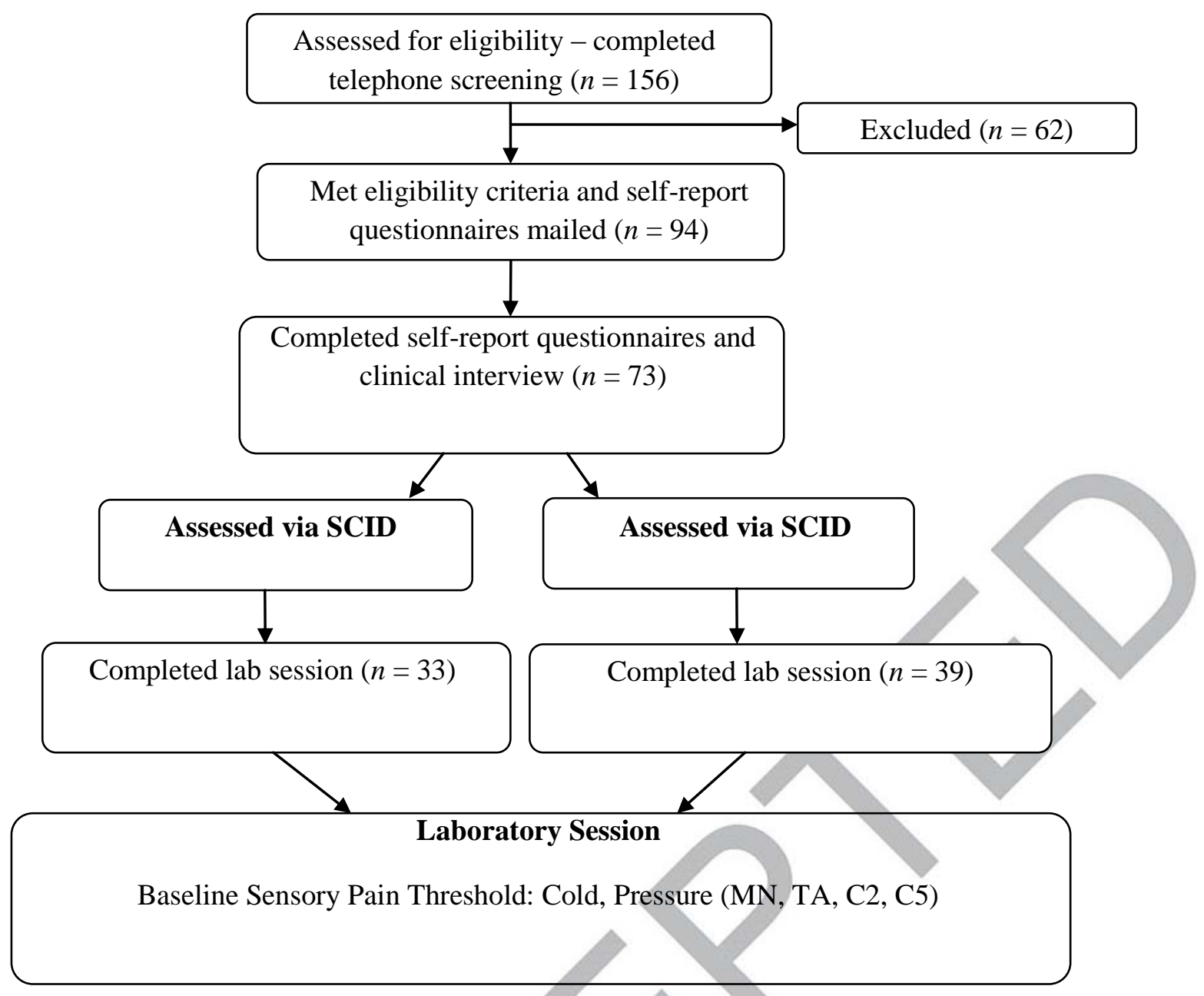

Note $. \mathrm{MN}=$ Median Nerve; $\mathrm{TA}=$ Tibialis Anterior; $\mathrm{C} 2=$ second cervical spinous process; $\mathrm{C} 5$ = fifth cervical spinous process.

Figure 2 Means and Standard Errors for NRS Negative Affect of the PTSD and No PTSD Groups at Pre- and Post-Trauma Cue

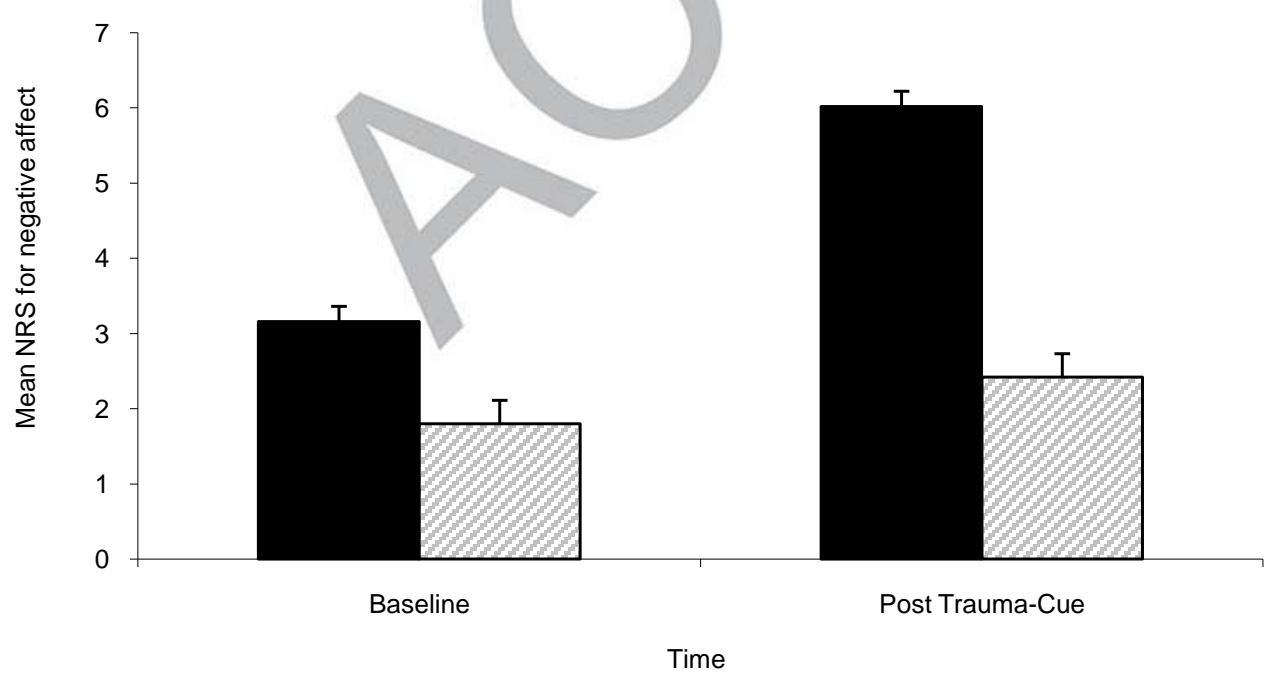


Figure3 Means and Standard Errors for NRS Pain of the PTSD and No PTSD Groups at Pre- and Post- Trauma Cue

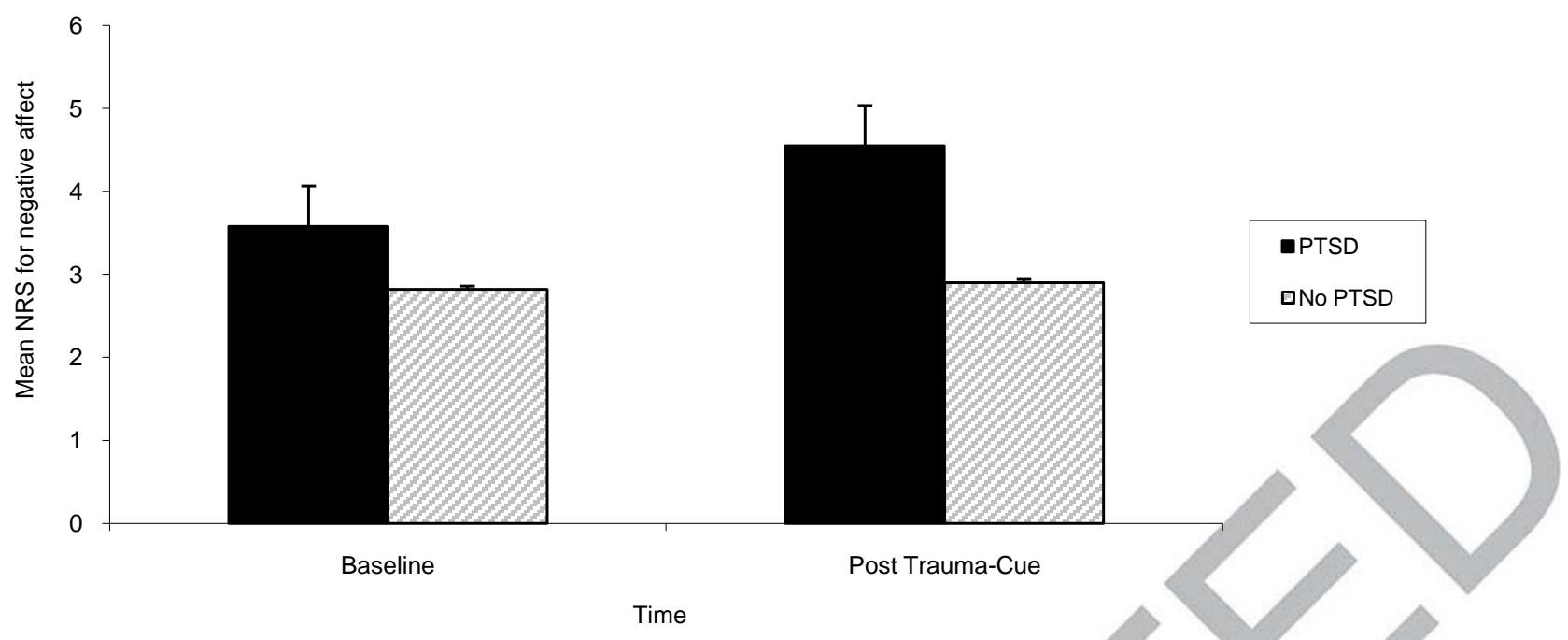

